\title{
Combining weak acids and fasting could be a viable strategy against infections by Ebola-like viruses
}

Yuhan Lin ${ }^{1}$, Lipeng Liao ${ }^{1}$, Chanyuk Lam ${ }^{1}$, Min Fu², Qiuyun Liu ${ }^{1}$ *

${ }^{1}$ School of Life Sciences, Sun Yat-Sen University, Guangzhou 510275, China.

${ }^{2}$ Deyang Health Committee, Sichuan, China

*Correspondence author: Qiuyun Liu, School of Life Sciences, Sun Yat-sen University, Guangzhou 510275, China.

E-mail address: lsslqy@ mail.sysu.edu.cn (Q. Liu) 


\begin{abstract}
Ebola-like viruses could depend on chloride or $\mathrm{HCl}$ to deliver lethal assault on human cells, causing hemorrhaging, bleeding, diarrhea, etc. It may harbor stressful peptides which can be generated in vivo after infections. Fasting with boiled rice water or with orange juice for a few days can halt virion production. However, infection-triggered cell lysis can produce nutrients for viral proliferation. The adoption of highly diluted weak organic acids such as acetic acid or lactic acid for smearing skin or foot bathing can neutralize chloride or $\mathrm{HCl}$ after absorption, and raise cellular $\mathrm{pH}$ to minimize cell lysis. Integration of fasting/fruit juice intake and highly diluted weak acids might reduce mortality rates of Ebola-like virus infections. The intake of $\mathrm{NaCl}$ should be minimized as it can give rise to chloride or $\mathrm{HCl}$ in cell microenvironment. Many food ingredients such as vinegar and sauce contain salt. Clinical trials must be conducted before the regimen is available for use.
\end{abstract}

Keywords

Ebola-like viruses; chloride; $\mathrm{HCl}$; fasting; weak acids 


\section{Conflict of interest}

None declared.

\section{ACKNOWLEDGMENT}

This work was supported by the Science and Technology Transformation Program of Sun Yat-sen University of China (33000-18843234) and Guangzhou Science and Technology Program (201804010328) to Q. Liu.

\section{REFERENCES}

1. Gan T, Fu M, Wu J, Wen L, Liu Q. (2018). How to design carbohydrate diet regimens for heart disease patients. Eur J Prev Cardiol 25:979-980.

2. Xing M, Li X, An S, Lan C, Fu M, Shi Y, et al. (2021). Anticancer nanometre-biomissiles carrying a leucine insulator and an antibody complementarity-determining region. Curr Sci 120:177-185.

3. An S, Li X, Tang M, Xing M, Zhang Y, Peng J, et al. (2020). The Role of acetate in the antagonization of oxalate: a potential causative molecule for heart disease and cancer death. Nat Prod Commun 15:1934578X2091369.

4. An S, Li X, Tang M, Yan S, Yang F, Zhu X, et al. (2019). Functional duality of ethanol on cancer. Med Hypotheses 122:124-125.

5. An S, Wan Y, Shi Y, Yang F, Liu Q. (2018). How to choose medicinally more valuable yogurt products for the prevention of heart disease and colorectal cancer. Eur J Prev Cardiol 25:2013-2014.

6. An S, Zhang X, Shi Y, Zhang J, Wan Y, Wang Y, et al. (2020). High glycine content in TDP-43 - A potential culprit for Limbic-predominant age-related TDP-43 encephalopathy. J Int Med Res 48:300060520929853.

7. Shi Y, An S, Wan Y, Yang F, Liu Q. (2019). How to best use acetic acid for the prevention of heart disease and cancer. Eur J Prev Cardiol 26:437-438.

8. Wan Y, Yan S, Zhang Y, An S, Yang K, Xu H, et al. (2020). The Pneumonia Outbreak: High isoleucine and high valine plus glycine contents are features of the proteins of COVID-19virus. Preprints 2020020289. doi: 10.20944/preprints202002.0289.v5.

9. Wan Y, Yan S, Zhang Y, Liu Q. (2020). Why COVID-19 virus is so deadly to cancer patients? Eur J Cancer Prev 29:365.

10. Zhang X, Ma X, Gan T, Shi Y, Wang Y, Liu Q. (2020). Secondary chemical bonding between insoluble calcium oxalate and carbonyl oxygen atoms of GLY and VAL residues triggers formation of A $\beta$ aggregates and their deposition in the brain. ACS Chem Neurosci 11:4007-4011.

11. Wan Y, Zhang J, Li X, Wang Y, Liu Q. (2019). Cellular states and secondary chemical bonding: a biochemical view of major human diseases. Biochem insights 12:1178626419877846. 